\section{Tropical Journal of Pathology and Microbiology}

\title{
Epidemiology of Mycotic Infections: Experience From A Tertiary Care Center Of Uttarakhand, India
}

\author{
Mohanty A. ${ }^{1}$, Rohilla R. ${ }^{2 *}$, Meena S. ${ }^{3}$, Bora M. ${ }^{4}$, Singh A. ${ }^{5}$, Kaistha N. ${ }^{6}$, Gupta P. ${ }^{7}$ \\ DOI: https://doi.org/10.17511/jopm.2021.i03.01
}

${ }^{1}$ Aroop Mohanty, M.D, Assistant Professor, ${ }^{2 *}$ Ranjana Rohilla, Assistant professor, Department of Microbiology, Shri Guru Ram Rai Institute of Medical \& Health Sciences, Dehradun, Uttarakhand, India. ${ }^{3}$ Suneeta Meena, M.D, Assistant Professor, Department of Laboratory Medicine, All India Institute of Medical Sciences, Delhi, India. 4 Mamta Bora, Student M.Sc, Department of Medical Microbiology, Uttaranchal PG College of Biomedical Sciences and Hospital, Rishikesh, Uttarakhand, India. ${ }^{5}$ Anshu Singh, Ph.D. scholar, ${ }^{6}$ Neelam Kaistha, M.D. Professor, 7 Pratima Gupta, M.D. Professor, $\mathbf{1 , 5 , 6 , 7}$ authors are affiliated with the Department of Microbiology, All India Institute of Medical Sciences, Rishikesh, Uttarakhand, India.

Introduction: The overall changing epidemiology of fungal infections in the current scenario is because of an increase in immunocompromised population including cancer patients, Human immunodeficiency virus (HIV)-infected patients, transplant receipts, and prolonged hospitalization with overuse of antimicrobial agents. These infections are challenging to diagnose and subsequently manage as their clinical symptomatology often mimics other common diseases like tuberculosis. Rapid diagnosis is limited and culture is often delayed due to slow growth rates of the causative agents. Objective: This is a retrospective study to know the spectrum and burden of mycotic infections in a tertiary care hospital. Methods: All samples collected from clinically suspected cases of fungal infections were sent to the Microbiology department over one year. The common specimens received were respiratory samples, scrapings from cornea, skin, and nail. All samples were first observed under direct microscopy using Potassium hydroxide $(\mathrm{KOH})$ examination for the presence of fungal elements and Gram stain for yeasts. India Ink examination was performed for sterile fluids. Fungal culture was done on Sabouraud's dextrose agar. Result: A total of 900 samples from various departments were included, $\mathrm{KOH}$ examination was positive for 380 samples (42\%) and fungal growth was obtained in 144 samples (16\%). Rare fungi like Trichosporon dohaense (blood culture), Cladophialophora bantiana (brain abscess), Scedosporium apiospermum and Candida auris (blood culture) were also isolated. Conclusion: Similar studies are needed to estimate the actual burden of the fungal infections in tertiary care health facilities, to help decrease the morbidity and mortality associated with underdiagnosed mycotic infections.

Keywords: Mycotic infections, Fungal spectrum, Fungal culture, Rare fungi

Corresponding Author

Ranjana Rohilla, Assistant professor, Department of Microbiology, Shri Guru Ram Rai Institute of Medical \& Health Sciences, Dehradun, Uttarakhand, India. Email: ranjana86choudhary@gmail.com

\section{How to Cite this Article} To Browse

Mohanty A, Rohilla R, Meena S, Bora M, Singh A, Kaistha N, Gupta P. Epidemiology of Mycotic Infections: Experience From A Tertiary Care Center Of Uttarakhand, India. Trop J Pathol Microbiol. $2021 ; 7(3): 93-98$. Available From https://pathology.medresearch.in/index.php/jopm/ar ticle/view/525

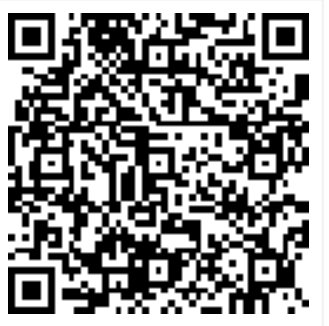

Manuscript Received 2021-04-20

Conflict of Interest No

Review Round 1
2021-04-30
Funding
Nil

Review Round 1 $\mathrm{Nil}$

Review Round 2
2021-05-07
Ethical Approval
Yes

Review Round 3

Plagiarism X-checker $6 \%$
Accepted 2021-05-13

(c) 2021 by Aroop Mohanty, Ranjana Rohilla, Suneeta Meena, Mamta Bora, Anshu Singh, Neelam Kaistha, Pratima Gupta and Published by Siddharth Health Research and Social Welfare Society. This is an Open Access article licensed under a Creative Commons Attribution 4.0 International License https://creativecommons.org/licenses/by/4.0/ unported [CC BY 4.0]. 


\section{Introduction}

Medical mycology has gained a lot of importance over recent years due to the emergence of new pathogenic fungi, including yeast and moulds [1]. The entire scenario regarding the epidemiology of fungal infections has undergone a paradigm shift. Earlier, the fungal isolates, which were mainly considered as non-pathogenic and laboratory contaminants, have now proved to be true pathogens and etiological agents of various emerging and opportunistic fungal infections. The overall increase in fungal infections is mainly due to the steep rise in population at risk, including cancer patients, transplant recipients, long term use of immunosuppressive agents, and prolonged hospitalization with overuse of broad-spectrum antibiotics and indwelling devices.

These organisms can also affect immunocompetent individuals [2-4]. Advances in medical science have helped clinicians treat fatal diseases, leading to prolonged life of immunocompromised individuals who in the further course get prone to opportunistic infections. Moreover, these infections are severe, rapidly progressing, and challenging to diagnose and treat as their clinical manifestations often mimic other common diseases. Rapid diagnosis is limited and culture is often delayed due to slow growth rates of many fungi. This study was undertaken to find the magnitude and incidence of fungal infections in our centre. This study is of particular relevance keeping in mind that our institute is a tertiary care health centre for people coming from hilly regions of the state where good quality health services are not approachable.

\section{Material and Methods}

This retrospective observational study has conducted by the Department of Microbiology at a tertiary level hospital situated in the Uttarakhand state of India from February 2019 to January 2020. All the samples collected from clinically suspected cases of fungal infections were sent to the Microbiology department (Mycology section). How the samples were collected and transported to the laboratory depending on the specimen type.

Samples received in leaky containers or sent in formalin were not accepted. The common specimens were respiratory samples like sputum, bronchoalveolar lavage (BAL) and scrapings from skin, cornea and nail.
All samples were first observed under direct microscopy for the presence of fungal elements by dissolving them in Potassium hydroxide $(\mathrm{KOH})$ solution (10\%-40\%) and for yeast cells by Gram's stain. India Ink examination was also performed for capsulated yeast cells. $40 \% \mathrm{KOH}$ was used for dissolving nail samples whereas $10 \%$ was used for all of the others (skin scrapings, cornea scrapings, respiratory samples, pus and hair clippings) $[5,6]$. After direct microscopy, all samples were put up for fungal culture. They were inoculated onto two culture Media; one of Sabouraud's dextrose agar (SDA) and the other being SDA with cycloheximide and chloramphenicol (Hi-Media, India). The culture tubes were incubated at $25^{\circ} \mathrm{C}$ and $37^{\circ} \mathrm{C}$, respectively and were examined twice a week for four weeks. Any growth obtained was further identified by colony morphology. Moulds were further identified by conidia formation seen in Lactophenol cotton blue mount (LPCB), slide culture and additional tests. The isolated yeast colonies were identified based on Gram staining, germ tube production, sporulation on cornmeal agar, urease production and colour production on CHROM agar [7-9]. All germ tube negative Candida spp. and few filamentous fungi were identified using Matrixassisted Laser Desorption/Ionization-time of flightMass spectrometry (MALDI-TOF-MS) (Bruker Daltonics, Germany). Approval was sought by the ethical and research committee of the institution. Data was entered and analyzed on Microsoft Excel and interpreted by descriptive methods in terms of frequency Distribution

\section{Results}

A total of 900 samples from patients admitted in various departments of the hospital were included in this study. Of all, 65\% (585) were from males and $35 \%$ (315) were from females. [Figure.1] Among these, $\mathrm{KOH}$ examination was positive for $42 \%$ (380) samples and fungal growth was obtained in $16 \%$ (144) samples while no growth was obtained in the rest of $84 \%$ (756) samples [Figure.2] In 26\% (236) of samples for which $\mathrm{KOH}$ examination was positive, growth was not observed. Plausible reasons for these findings could be inappropriateness of samples, samples retrieved after the start of antifungal therapy or inadequate temperature or culture conditions, mishandling of sample while inoculation of culture medium. The maximum number of infections were seen in the adult age group $[657(73 \%)]$ followed by the pediatric age group [172(19.11\%)]. 
Of all the samples received, skin, hair and nail samples accounted for the maximum percentage i.e., $25 \%$ (225), followed by sputum samples $21 \%$ (189), Cerebrospinal fluid (CSF) 16\% (144), bronchoalveolar lavage (BAL)15\% (135), corneal scrapings $12 \%$ (110), pus sample $7 \%$ (63) and tissue 4\% (34). [Figure 3]. Most of the culture positives were obtained from tissue samples (73.50\%, 25/34), followed by corneal scrapings (31\%, 34/110), BAL $(19.20 \%, 26 / 135)$, pus (14.30\%, 9/63), sputum (13.20\%, 25/189), skin, nail \& appendages $(10.60 \%, 24 / 225)$ and CSF $(0.69 \%, 1 / 144)$.

[Table 1, Figure 3]. Among the 144 culture-positive samples, the predominant fungus isolated was Candida albicans (40.3\%, 58/144), followed by filamentous fungi like Aspergillus spp. (16\%, 23/144), Fusarium spp (13.2\%, 19/144), and Zygomycetes (10.4\%, 15/144). Rare fungi like Trichosporon dohaense, Scedosporium apiospermum and Cladophialophora bantiana were also isolated. [Table 1]

Although fungal infections are not as common as bacterial, viral, and parasitic infections as causes of human suffering, they still are the most challenging diseases to be managed. In recent times, mycotic infections are noticeably on the rise in health care facilities. Still, the data on the burden of these infections in our country is under-reported, though the country's climate is suitable for rising fungal infections.

There are very few established reference mycology laboratories in the country and the clinicians are still not aware of the change in mycotic infections spectra. [10]. Consequently, there is a scarcity of studies mentioning the spectrum and aetiology of fungal infections.

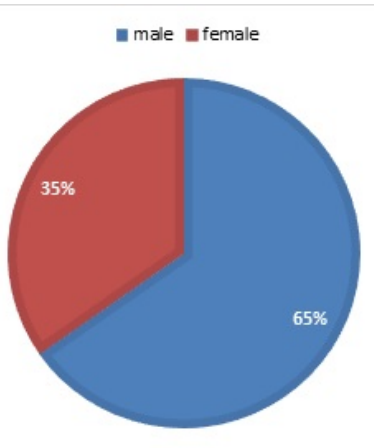

Figure 1: Sex distribution of total samples

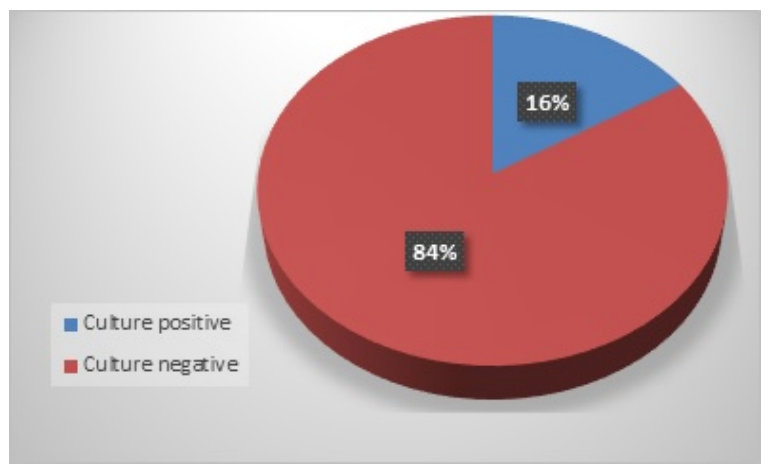

Figure 2: Distribution of total samples

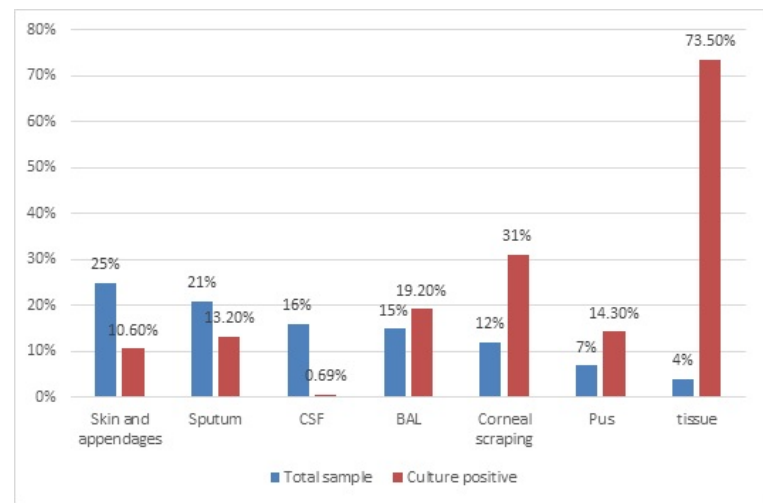

Figure 3: Distribution of total and culturepositive samples

Table 1: Spectrum of isolates among various samples

\begin{tabular}{|c|c|c|c|c|c|c|c|c|c|}
\hline Organism & BAL & Sputum & Nail & Skin & CSF \& blood & Pus & Tissue & Eye & Total \\
\hline c. Albicans & 22 & 22 & & & & 3 & 1 & & 48 \\
\hline Non-albicans Candida & 6 & 3 & & & 1 & & & & 10 \\
\hline A. fumigatus & 1 & 1 & & & & & 1 & & 3 \\
\hline A. flavus & 1 & 1 & & & & 1 & 7 & 7 & 17 \\
\hline A. niger & 1 & 1 & & & & & 1 & & 3 \\
\hline T. asahii & 1 & & & & & & 1 & & 2 \\
\hline T. mentagrophytes & & & 3 & 2 & & & & & 5 \\
\hline T. rubrum & & & 1 & 1 & & & & & 2 \\
\hline Malassezia spp. & & & & 10 & & & & & 8 \\
\hline
\end{tabular}




\begin{tabular}{|l|l|l|l|l|l|l|l|l|l|}
\hline Fusarium & & & & & & & & 19 & 19 \\
\hline Rhizopus & & & & & & 4 & 6 & & 10 \\
\hline Rhizomucor & & & & & & & 2 & & 2 \\
\hline Mucor & & & & & & & 3 & & 3 \\
\hline Alternaria & & & & & & & 3 & & 3 \\
\hline Curvularia & & & & & & & & 4 & 4 \\
\hline Geotrichum & & & & & & & & 1 & 1 \\
\hline P. boydii & & & & & & & & 1 & 1 \\
\hline Cryptococcus & & & & & 1 & & & & 1 \\
\hline Total & 26 & 25 & 6 & 18 & 1 & 9 & 25 & 34 & 144 \\
\hline
\end{tabular}

\section{Discussion}

A total of 900 samples were received from various suspected cases from all the Out-patient Departments (OPDs) and wards, in the Mycology laboratory. Of these, 144 (16\%) were culture positive. A study from Western India documented a culture positivity rate of $48 \%$, while a study from the Northern part reported it to be $14 \%$. [11, 12]. Candida was the commonest isolate followed by filamentous fungi like Aspergillus spp., Fusarium spp, and Zygomycetes. [13]. Few cases of Cryptococcus spp. were also isolated. [14-16]. The lesser percentage of culture positivity is attributed to the fact that technical staff was posted on a rotation basis in different sections of the Microbiology Department owing to an acute shortage of workforce in the institute.

There was a high rate of attrition concerning those handling specimens sent for fungal culture. A wide variation was observed during other months concerning fungal growth obtained in cultures. In due course of time, an increasing rate of culture positivity was observed indicating streamlining of laboratory procedures and workflow. Clinical Microbiology has a well-earned reputation for unpredictability \& the Mycology section advocates this dictum. $[17,18]$. Most of the culture positives were obtained from tissue samples and corneal scrapings followed by respiratory samples, skin \& appendages, pus and CSF. The ocular mycology section was developed as 'Eye bank' and cornea transplant facilities started at the institute in the year 2019. Fungal growth was obtained in $31 \%$ (34/110) corneal scrapings sent to the ocular mycology section. $[19,20]$. Rare fungi like Trichosporon (blood culture), Cladophialophora bantiana (C. bantiana) (brain abscess), and Scedosporium apiospermum (corneal scraping), Wickerhamomyces anomalous (blood) were also isolated.
T. dohaense was isolated from a case of T-cell acute lymphoblastic leukemia (T-ALL) on hyper CVAD/MC (hyper fractionated- cyclophosphamide, vincristine, doxorubicin and dexamethasone/methotrexate) chemotherapy. Patient succumbed to sepsis and multiorgan failure owing to the highly invasive fungal infection. [21-23] C. bantiana was isolated from an immunocompetent individual diagnosed to be a case of brain abscess post radical surgical intervention. Amphotericin B and voriconazole were added to the treatment.

But she gradually developed multiorgan failure and succumbed to the fatal infection. The timely diagnosis of these rare agents can prove to be pivotal in the management of the patient. Commoner isolates with otherwise grave and protean manifestations, with high morbidity and mortality, were also isolated. An immunocompetent male was diagnosed with angio-invasive cerebral aspergillosis, managed with right frontal craniotomy and voriconazole was continued. The Patient was well in nine months' follow-up and an imaging scan showed complete resolution of the lesion. Another male with uncontrolled diabetes was diagnosed with Curvularia lunata rhinosinusitis with orbital cellulitis. Imaging was indicative of orbital extension.

The Patient was subjected to extensive surgical debridement, along with antifungals. Rhinosinusitis was resolved; however, the loss of vision was irreparable. The other opportunistic infections because of Mucorales, Cryptococcus, and Non Albicans Candida were also reported. C. Auris was also isolated from the blood culture of a young female who underwent a Whipple's procedure for the carcinoma head of the pancreas. She was successfully treated with caspofungin.

The scope of tests was also expanded to include proteonomics-based MALDI-TOF-MS technique and calcofluor white staining. [24-26]. 
This is a preliminary hospital-based study to know the magnitude of the repertoire of fungal infections in the hospital and to identify the varied etiological agents. More detailed multicentric prospective studies considering the demographic data, risk factors, occupation, and socioeconomic condition of the Patient can be conducted.

\section{Conclusion}

To summarize, similar studies backed up by more comprehensive laboratory investigations, including serological tests, exoantigen tests and molecular methods, are needed to estimate the actual burden of fungal infections in tertiary care health facilities. Improved diagnostic and therapeutic strategies, evaluation of risk factors, and the development of new methods for rapid diagnosis and monitoring should help decrease the morbidity and mortality associated with mycotic infections.

\section{What does this study add to existing knowledge?}

There is a paucity of literature on this topic and this will provide a basic idea about the prevalence of fungal pathogens in a hospital setting. Besides the use of MALDI-TOF-MS has revolutionized the identification of rare fungi including moulds.

\section{Author contribution}

Dr. Aroop Mohanty -first draft and data analysis, Dr. Ranjana Rohilla - concept, Dr. Suneeta Meena- data analysis, Mamta Bora-images, Anshu Singh- revision of the manuscript, Dr. Neelam Kaistha-data analysis, literature search and Dr. Pratima Guptaliterature search.

\section{Reference}

01. Kashyap B, Das S, Kaur I R, Jhamb R, Jain S, Singal A, Gupta N. Fungal profile of clinical specimens from a tertiary care hospital. Asian Pacific Journal of Tropical Biomedicine. 2012;2(1)S401-S405. [Crossref][PubMed][Google Scholar]

02. Richardson MD. Changing patterns and trends in systemic fungal infections. J Antimicrob Chemother. 2005 Sep;56 Suppl 1;i5-i11. doi: 10.1093/jac/dki218 [Crossref][PubMed][Google Scholar]
03. Shrihari N, Kumudini TS, Mariraj J and Krishna S. The Prevalence of Keratomycosis, Dermatophytosis and Onychomycosis in a Tertiary care Hospital. International Journal of Medical and Health Science. 2012;1;25-30. [Crossref][PubMed] [Google Scholar]

04. Husain S, Alexander BD, Munoz P, Avery RK, Houston $S$, Pruett $T$, Jacobs $R$, Dominguez $E A$, Tollemar JG, Baumgarten K, Yu CM, Wagener MM, Linden $P$, Kusne $S$, Singh N. Opportunistic mycelial fungal infections in organ transplant recipientsemerging importance of non-Aspergillus mycelial fungi. Clin Infect Dis. 2003 Jul 15;37(2)221-9. doi: 10.1086/375822 [Crossref][PubMed][Google Scholar]

05. Cheesbrough, Monica. District laboratory practice in tropical countries, part 2. Cambridge university press. 2005. [Crossref][PubMed][Google Scholar]

06. Collee JG, Fraser AG, Marmion BP, Simmons A. Mackie \& McCartney Practical Medical Microbiology. 14th ed, New York- Churchill Livingstone Publication. 1996;p-695-717. [Crossref][PubMed] [Google Scholar]

07. Glenn DR, Norman LG. Laboratory diagnosis, InTopley and Wilsons Medical Mycology. 10th ed, London- Arnold Publications. 2005;82-9. [Crossref] [PubMed][Google Scholar]

08. Koneman EW, Allen SD, Janda WM, Schreckenberger PC, Winn WC Jr. Koneman's Colour Atlas and Textbook of Diagnostic Microbiology. 6th ed, Philadelphia- Lippincott Williams and Wilkin's. 2006;1156-246. [Crossref][PubMed][Google Scholar]

09. Agarwal S, Manchanda V, Verma N, Bhalla P. Yeast identification in routine clinical microbiology laboratory and its clinical relevance. Indian $\mathrm{J}$ Med Microbiol. 2011 Apr-Jun;29(2)172-7. doi: [Article] [Crossref][PubMed][Google Scholar]

10. Chakrabarti A, Chatterjee SS, Shivaprakash MR. Overview of opportunistic fungal infections in India. Nihon Ishinkin Gakkai Zasshi. 2008;49(3)165-72. doi: 10.3314/jjmm.49.165 [Crossref][PubMed] [Google Scholar]

11. Mittal M, Sharma V, Gupta V. Isolation of fungus from various clinical specimens in patients of a tertiary care hospital of North India. JMSCR. 2019;7(4)753-6. doi: [Article][Crossref][PubMed] [Google Scholar] 
12. Singh V, Mohanty A, Narain TA, Kaistha N, Gupta $P$, Anshu. Candida tropicalis in the tropics- $A$ rare fungal cause of perinephric abscess. J Family Med Prim Care. 2020 Sep 30;9(9)5062-5065. doi: 10.4103/jfmpc.jfmpc_784_20 [Crossref][PubMed] [Google Scholar]

13. Jha MK, Mohanty A, Gupta P. Cryptococcus gattii meningitis in a diabetic adult in South India. J Family Med Prim Care. 2019 Mar;8(3)1253-1256. doi: 10.4103/jfmpc.jfmpc_66_19 [Crossref] [PubMed][Google Scholar]

14. Mohanty A, Bhatia M, Kabi A, Chatterjee K, Kaistha N, Omar BJ, Gupta PK, Gupta P. Cryptococcal meningitis- An under-reported disease from the hills of Uttarakhand- A hospital-based cross-sectional study. J Family Med Prim Care. 2019 Jun;8(6)2008-2011.

doi: 10.4103/jfmpc.jfmpc_216_19 [Crossref][PubMed] [Google Scholar]

15. Kabi A, Mohanty A, Kumar S, Jha M, Gupta P. Rare case of chronic meningitis by cryptococcus neoformans var, grubii in an immunocompetent host. Int J Scientific Research. 2020;9(07)17-18. [Crossref][PubMed][Google Scholar]

16. Rhoads DD, Sintchenko $V$, Rauch CA, Pantanowitz L. Clinical microbiology informatics. Clin Microbiol Rev. 2014 Oct;27(4)1025-47. doi: 10.1128/CMR.00049-14 [Crossref][PubMed][Google Scholar]

17. Mohanty A, Meena S, Nath $U$ K, Vaniyath $S$, Kaistha N. Candida krusei Infection in an Acute Lymphocytic Leukaemia Patient- A Case Report. Journal of Clinical \& Diagnostic Research. 2020;14(5). [Crossref][PubMed][Google Scholar]

18. Rohilla R, Meena S, Gupta N, Mohanty A, Kaistha N, Gupta P, Mangla A, Rekha US, Omar BJ. Clinico-etiological profile of microbial keratitis- $A$ pilot study from the foothills of Himalayas. Indian J Ophthalmol. 2020 Oct;68(10)2323-2324. doi: 10.4103/ijo.IJO_2125_19 [Crossref][PubMed] [Google Scholar]

19. Rohilla R, Meena S, Mohanty A, Gupta N, Kaistha N, Gupta P, Mangla A, Singh A. Etiological spectrum of infectious keratitis in the era of MALDITOF-MS at a tertiary care hospital. J Family Med Prim Care. 2020 Sep 30;9(9)4576-4581. doi: 10.4103/jfmpc.jfmpc_630_20 [Crossref][PubMed] [Google Scholar]
20. Mohanty A, Meena S, Nath UK, Bakliwal A, Kaistha N, Gupta P. Trichosporon dohaense causing life threatening fungemia in acute leukemia- First case report from India. Indian J Pathol Microbiol. 2020. [Crossref][PubMed][Google Scholar]

21. Tyagi A K, Suji P S, Kumar A, Varshney S, Mohanty A, Gupta P. First Report on Concomitant Infection of Nasal Myiasis and Trichosporonosis in an Uncontrolled Diabetic Patient- Case Report. Indian Journal of Otolaryngology and Head \& Neck Surgery. 2020;1-3. [Crossref][PubMed][Google Scholar]

22. Mehta V, Mohanty A, Meena S, Rahul JS, Uttam Kumar N, Chattopadhyay D, Bakliwal A, Choudhary R, Gupta P. Wickerhamomyces anomalous- A Rare Cause of Fungemia Causing Febrile Neutropenia in Acute Lymphoblastic Leukemia. Case Rep Infect Dis. 2020 Dec 29;2020;8847853. doi: 10.1155/2020/8847853 [Crossref][PubMed][Google Scholar]

23. Goyal N, Narula H, Chaturvedi J, Agrawal S, Dash C, Meena S, Kaistha N. Angio-invasive Cerebral Aspergillosis Resulting in Hemispheric Infarct in an Immunocompetent Man. Med Mycol J. 2020;61(3)49-53. doi: 10.3314/mmj.20-00005 [Crossref][PubMed][Google Scholar]

24. Narula H, Meena S, Jha S, Kaistha N, Pathania M, Gupta P. Curvularia lunata causing orbital cellulitis in a diabetic patient- An old fungus in a new territory. Curr Med Mycol. 2020;6(1)51-54. doi: 10.18502/cmm.6.1.2510 [Crossref][PubMed] [Google Scholar]

25. Meena S, Rohilla R, Kaistha N, Singh A, Gupta P. Candida auris emergence in the Himalayan foothillsFirst case report from Uttarakhand, India. Curr Med Mycol. 2020;6(1)47-50. doi: 10.18502/cmm.6.1.2509 [Crossref][PubMed] [Google Scholar] 